\title{
Callum, Walker; Federici, Federico (Eds.). Eye Tracking AND MULTIDISCIPLINARY StUdIES ON TRANSLATION. AMSTERDAM, Benjamins Publishing Company, 2018, 299 PP., ISBN: 978-9- 0272-0169-0.
}

Eye tracking is "the process of recording the point of gaze of a person and the movement of the eyes from one point to another" (Saldanha \& O'Brien, 2013, p. 136). In recent years, eye-tracking approaches, "borrowed" from cognitive and psychological sciences, have attracted considerable interest in Translation Studies (Doherty, 2016). The decrease in cost of eye trackers, improved handling, and the creation of more one-stop-shop analysis software packages have all contributed to the development of this research avenue in Translation Studies (Schulte-Mecklenbeck et al., 2017).

Eye Tracking and Multidisciplinary Studies on Translation, edited by Walker and Federici, brings together a number of contributions regarding the use of eye tracking in Translation Studies. This book aims to serve both as "a methodological frame of reference for new and seasoned researchers alike on the tools, methods and approaches adopted [in eye tracking research] [...] and the principles that are currently prevalent in this discipline" (p. 1). In particular, it is intended to act as a foundation for researchers who are working in emergent Cognitive Translation Studies. Readers are encouraged to adopt a critical eye in order to compare and contrast the suggested methods as the contributors embark on their research using a methodology with a number of inherent challenges ranging from experimental design to data collection and analysis. This volume, which consists of 299 pages divided into 12 chapters, includes an introductory part, with the main body of the text then divided into three sections: method, process, and products.

The introductory section to this volume consists of two chapters in which the principles of eye tracking research in Translation Studies are examined by the editors. They also call on scholars in Translation Studies to update not only their experimental and theoretical skills, but also their methods of statistical analysis and presentation in line with recent developments in Psychology. Contributions to the first of the three sections noted in the previous paragraph present methodological challenges (Chapters 3-5) and focus on experimental design and data analysis. The second section deals with research into the translation process (Chapters 69), and the third with Product Reception Studies (Chapters 10-12), areas that have hitherto received little attention. 
In Section I (Addressing the Methods), Teixeira and O'Brien shed light on the use of eye tracking for collecting data from a concrete experiment which observes the interaction between professional translators and the graphical interfaces of computer-assisted translation (CAT) tools in the physical workplace. Specifically, the primary considerations and obstacles presented in this chapter include eye tracker selection, participant enlistment, research equipment and software setup, eye-tracking software configuration, data collection, data analysis, tool synchronisation, recordings segmentation, and technical considerations involving the use of monocular versus binocular data. Their valuable concerns provide an exceptional methodological framework for future studies carried out under similar conditions. Moorkens explores the use of eye tracking as a measurement of cognitive effort for post-editing machine translation, building on Kring's (2001) measurements of post-editing effort (temporal, technical, and cognitive). The study argues that two main eye-tracking measurements can be used as indicators for cognitive effort, namely fixation count and fixation duration. They conclude by calling for a mixed-methods approach (using quantitative and qualitative data) to post-editing studies, which could be a worthwhile means to add new insights for future eye-tracking studies concerning user interaction with machine translation. Doherty emphasises the methodological limitations of eye tracking in the translation process and product studies, namely, the approach to statistical analysis. He advocates the application of regression designs rather than factorial designs in order to better analyse the relationships between variables within eye-tracking data. $\mathrm{He}$ also promotes the use of rigorous statistical methods for time-course data, i.e. growth curve modelling instead of the more typical analyses of variance (ANOVA) - a collection of statistical models and their associated estimation procedures (such as the "variation" among and between groups) used to analyse the statistical significance of mean differences (Tabachnick \& Fidell, 2006). He ends his contribution with a call for more robust training in research methods for researchers working in Cognitive Translation Studies. These arguments will provide a sound foundation to explore novel, rigorous methods of statistical analysis that are well established in fields like the Cognitive and Psychological Sciences but have not yet permeated thoroughly into the cognitive paradigms of Translation Studies.

In Section II (Targeting the Process Studies), Martínez-Gómez, Han, Carl and Aizawa present a framework for recognising and characterising the translator's attributes and activity patterns, correlating these attributes with translation activities from eye tracking and keystroke logging data to suggest ideas for modelling the translation process in order to develop more effective CAT tools. Their data results (using ANOVA methods) demonstrate that the combination of sequences of keystrokes and fixations could be of great 
benefit to the study of translation behaviour, and that faze modality plays an essential role in modelling translator emotional state, expertise, and experience. This research is of considerable inspiration to the development of more accurate models for the translation process and the design of more effective interfaces for CAT tools. Schmaltz examines the cognitive effort guiding decision making in the process of translating linguistic metaphors from Chinese into Portuguese. To triangulate the results, key-logging and eye-tracking data, as well as retrospective verbal protocols and questionnaires, are used during the data collection process. The statistical analysis adopts mixed-effects models. The study finds that, firstly, there is no expression-type (metaphorical or literal) impact on total production time, and, secondly, the predictor translation strategy is relevant to production time. This study contributes to translation research into the Chinese/Portuguese metaphor, translation pedagogy, and more generally, research into the translation process itself so as to better understand the complexity of cognitive processes involved in the translation of metaphors. Huang presents an analysis on the working styles of student translators who use both their mental as well as physical capacities to perform three revision tasks: self-revision, peer-revision, and post-editing. She triangulates eye tracking, keystrokes, and cue-based retrospection data in a Chinese/English language-pair experiment. By comparing students' cognitive processes within and across tasks, the study finds three types of reviser: habit-oriented, task-oriented, and habit/task-oriented. By presenting the performance of the student translator's working styles alongside those of the professionals in existing literature, this research offers an insight into the trainee translator's behaviour when involved in revision tasks and, therefore, acts as a significant contribution to translation pedagogy. Szarkowska, Dutka, Szychowska, and Pilipczuk look at the little explored field of respeaking- a method used to produce real-time subtitles for live television programmes, especially for the $d /$ Deaf or hard-of-hearing. Their study utilises eye tracking to record the visual attention of the participants (divided into groups: interpreters, translators, subtitlers, and the control group) during an intralingual respeaking task (Polish to Polish) and a short proof-reading task. The contributors examine the number of fixations and mean fixation duration on major screen areas: picture, subtitles, dictation area and subtitle panel. The results demonstrate that those with subtitling experience can manage their visual attention most efficiently, as more often they fixated on key screen areas-the picture and the subtitles. They also performed best in the proof-reading task, as they fixated more often on the subtitles and the subtitle panel in comparison to other groups. Their findings not only reveal visual attention distribution during a respeaking task but also show that 
those with subtitling experience make them more suitable candidates for training as respeakers.

Finally, in Section III (Mapping the Product Reception Research), Filizzola employs eye tracking and online survey questionnaires to tap into Italians' perception and reception of British stand-up comedy with interlingual subtitles. The study finds that: firstly, a greater focus on subtitles does not undermine participants' enjoyment of stand-up comedy and, secondly, English proficiency and familiarity with watching English subtitled AVT products do not detract from viewers' appreciation either. This research offers an insightful contribution to the broader question of the effects of interlingual AVT products on the viewing experience of audiences-an area of research that is still nascent in Translation Studies. Walker undertakes a novel venture into an almost entirely unexplored area-equivalent effect and cognitive equivalence. He utilises eye tracking to compare readers' cognitive efforts in three different translated versions of the same text. This quantitative means to appraise cognitive equivalence can be combined with qualitative methods to explore the differing perceptions of marked language in literature before and after translation and, therefore, makes a significant contribution to the ongoing discourse on equivalence in Translation Studies. Given the originality of this approach, there is considerable scope for additional studies employing this type of methodology. This chapter proposes a methodological framework that can be adapted and tested by other researchers, using different case studies and various experimental conditions. Łabendowicz investigates the impact of AVT modalities (subtitling, dubbing, and voice-over) on viewers' reception and perception. She applies eye tracking and pre- and post-interviews to address audience responses to translated forms of humorous, source-culture-embedded audiovisual materials. Her study finds that the voiced-over or dubbed versions are more likely to be considered funny than are subtitled versions. This may be because participants can more easily immerse themselves in the content. However, this does not necessarily contribute to the understanding of the cultural references featured. The findings of this chapter provide a compelling insight into the impact of various modes of AVT on audience reception and will likely inspire future studies in this emerging field.

As noted by the editors, "the experimental method presented in this volume is not [...] 'ideal'" (p. 253), and some aspects could be optimised in future experiments, such as experiment design and statistical analysis. Personally, I consider there are certain limitations to eye-tracking studies; for example, a fixation cannot always be interpreted as information processing, and the absence of a fixation cannot necessarily be interpreted as the 
absence of processing, as eye trackers do not measure the use of peripheral vision. This is also explained in Jakobsen's (2014, p. 74) "dog-on-a-leash" theory. To overcome this shortcoming, it would be better to use additional biosensors, such as electroencephalogram (EEG), event-related brain potential (ERP), and functional magnetic resonance imaging (fMRI) to test participants' brain activation/activity before analysing eye reactions to the stimuli in order to validate results and the corresponding conclusions. Regarding statistical analysis, more rigorous analysis models, such as linear mixed models, could be used to analyse the finalised statistics in order to take in as much information as possible, instead of using the more traditional ANOVA statistical methods, which could lead to a reduction of data and loss of information under certain circumstances. Apart from SPSS, other software packages, such as $\mathrm{R}$ or Python, could also be applied as tools for data analysis to enrich the experimental protocol.

Although a number of improvements have been mentioned in the previous paragraph, this collective volume is a fantastic example of a sensibly coordinated academic publication, combining kaleidoscopic methodologies and experiments on eye-tracking. Eye-tracking methodologies can be applied to various areas within Translation Studies in a range of languages and contexts-be they on methods, process, or reception issues - which, as posited by the authors of this collective book, are expected to become ever-more important in Translation Studies to fully embrace and consolidate its interdisciplinarity.

\section{REFERENCES}

Doherty, S. (2016). The impact of translation technologies on the process and product of translation. International Journal of Communication, 10, 947-969.

Jakobsen, A. L. (2014). The development and current state of translation process research. In B. Elke, M. Reine and D. Luc van (Eds.), The Known Unknowns of Translation Studies (pp. 65-88). Amsterdam, Netherlands: John Benjamins Publishing Company.

Krings, H. P. (2001). Repairing Texts: Empirical Investigations of Machine Translation Post-Editing Processes. Ohio, US: The Kent State University Press.

Saldanha, G., \& O'Brien, S. (2013). Research Methodologies in Translation Studies. Manchester, UK: St. Jerome Publishing. 
Schulte-Mecklenbeck, M., Johnson, J. G., Böckenholt, U., Goldstein, D. G., Russo, J. E., Sullivan, N. J., \& Willemsen, M. C. (2017). Processtracing methods in decision making: On growing up in the 70s. Current Directions in Psychological Science, 26(5), 442-450.

DOI: $10.1177 / 0963721417708229$

Tabachnick, B. G., \& Fidell, L. S. (2006). Experimental Designs Using ANOVA. Belmont, CA: Duxbury Press.

[JIA HUIHUANG] 